\title{
COMMUNICATION
}

\section{Avulsions dentaires dans une maladie de Rendu Osler ayant préalablement nécessité une embolisation et une intervention de Young}

\section{Malthiéry E, Favre de Thierrens C, Fauroux MA, Levallois B, Torres JH}

Service d'Odontologie, Département de Médecine Orale Chirurgie Orale, CHRU Montpellier

La maladie de Rendu Osler (MRO) est une dysplasie vasculaire génétique. Son diagnostic, d'abord clinique, repose sur la triade «épistaxis récidivantes, télangiectasies cutanéomuqueuses et viscérales, hérédité autosomique dominante » (1). La MRO doit être connue de l'odontologiste qui peut participer à son diagnostic d'une part, et d'autre part doit proposer une prise en charge adaptée. Elle provoque en effet des manifestations hémorragiques, des télangiectasies cutanéo-muqueuses et des shunts vasculaires viscéraux, parfois eux-mêmes à l'origine d'abcès cérébraux après avulsion dentaire (2). Les épistaxis graves peuvent être traitées par ligature vasculaire ou embolisation sélective. Dans de rares cas, la prise en charge peut même aller jusqu'à l'intervention de Young : après incision circonférentielle de la jonction cutanéo-muqueuse du vestibule nasal antérieur, 3 lambeaux sont réalisés, déplacés et suturés pour fermer chirurgicalement les narines (3).

En février 2015, un patient de 58 ans a consulté au Service d'Odontologie du CHRU pour des mobilités dentaires et des saignements gingivaux. II présentait une maladie de Rendu Osler, découverte à l'âge de 35 ans, suite à des épistaxis répétées. Cette pathologie était traitée médicalement par de l'acide tranexamique ( $1 \mathrm{~g}$ matin, midi et soir). Au plan chirurgical, il avait eu une embolisation bilatérale dans le territoire de l'artère faciale en 2010, ainsi qu'une opération de Young en 2013. Malgré ces interventions, il avait été hospitalisé à plusieurs reprises pour des épistaxis importantes, nécessitant des perfusions de fer. L'examen clinique oral a révélé une parodontite chronique généralisée nécessitant l'avulsion de quatre dents (16, 15, 25 \& 26). L'intervention a été menée au bloc opératoire de l'Unité de Chirurgie Ambulatoire du CHRU, après avoir prévenu le radiologue interventionniste, sous anesthésie locale et sédation intraveineuse, avec mise en place de gaze de collagène hémostatique dans les alvéoles, sutures par points séparés et colle biologique. Des compressions locales à l'acide tranexamique ont été prescrites. Aucun saignement post opératoire n'a été rapporté et le contrôle à 14 jours a montré une bonne cicatrisation.

Ce cas rappelle que la prise en charge des patients atteints de MRO demande des mesures d'hémostase rigoureuses et au minimum une surveillance post opératoire ambulatoire. Par ailleurs, il fournit une imagerie remarquable, avec notamment une radiographie panoramique gardant les traces de l'embolisation, en soulignant très nettement les trajets artériels. De même, l'imagerie 3D objective la fermeture antérieure des fosses nasales, conséquence de l'intervention de Young.

\section{Références}

1 - Duffau P. et al., Rev Med Interne 2014,35:21-27.

2 - Shovlin C et al., Br Dent J. 2008,205:531-533.

3 - Richer SL et al., Am J Allergy 2012,26:401-404.

This is an Open Access article distributed under the terms of the Creative Commons Attribution License 4.0, which permits unrestricted use, distribution, and reproduction in any medium, provided the original work is properly cited. 